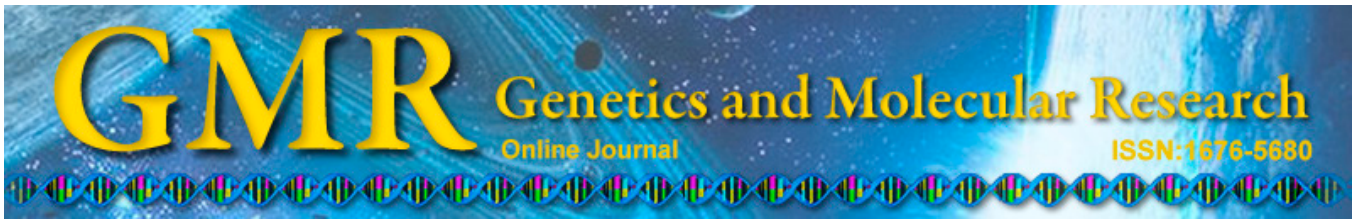

\title{
Molecular characterization of interferon regulatory factor 1 in Bubalus bubalis
}

\author{
N.B. Stafuzza, M.M. Borges and M.E.J. Amaral-Trusty \\ Departamento de Biologia, Instituto de Biociências, Letras e Ciências Exatas, \\ Universidade Estadual Paulista, São José do Rio Preto, SP, Brasil \\ Corresponding author: M.E.J. Amaral-Trusty \\ E-mail: eamaral@ibilce.unesp.br \\ Genet. Mol. Res. 14 (3): 10919-10928 (2015) \\ Received February 25, 2015 \\ Accepted June 6, 2015 \\ Published September 21, 2015 \\ DOI http://dx.doi.org/10.4238/2015.September.21.2
}

\begin{abstract}
Interferon regulatory factor 1 (IRF1) is functionally diverse in the regulation of immune response and is considered to be an important candidate gene for studying disease susceptibility in mammals. In this paper, we characterized the whole sequence of the IRF1 gene in river buffalo (Bubalus bubalis) and compared genomic and the amino acid sequences between different species. The buffalo IRF1 gene was 7099 bp long and organized into 10 exons and nine introns. Its molecular structure showed exactly the same number of exons (10) and introns (nine) in bovids, mice, horses, humans, and chickens. However, rats did not have exon 5, but had the largest exon 4 , which suggests that exon 5 was incorporated into exon 4 . The coding and the amino acid sequences of the gene showed that identity varied from 73 to $99 \%$ at the coding sequence level and from 61 to $100 \%$ at the amino acid level when compared with other mammals and chickens. Comparative analysis of the gene sequence between two different buffalo breeds, Murrah and Mediterranean, revealed six potential SNPs that are primarily located in the $5^{\prime}$ and $3^{\prime}$ UTRs.
\end{abstract}

Key words: BAC library; Interferon regulatory factor 1; Murrah; Pyrosequencing; River buffalo 


\section{INTRODUCTION}

The interferon-regulatory factor (IRF) gene family encodes transcription factors with multiple biological functions, including immune defense against virus and bacteria, stress response, cell differentiation, and reproduction as well as development of vertebrates (Ozato et al., 2007; Bazer et al., 2008; Tamura et al., 2008; Chen and Royer Jr, 2010). These factors regulate the expression of interferons and interferon-stimulated genes by binding to specific elements in their promoters (Mamane et al., 1999; Taniguchi et al., 2001).

In mammals, the IRF gene family is currently composed of nine members that are coded by phylogenetically related genes (IRF1 to IRF9) (Honda and Taniguchi, 2006; Nehyba et al., 2009). These genes share a high degree of similarity in genomic structure, which indicates that they could have evolved under a similar selective pressure in different classes of mammals (Fragale et al., 2013).

IRF1 is the most significant multifunctional factor and is involved in many aspects of the innate and adaptive immune responses, including hematopoietic differentiation, antiviral and antibacterial responses, cell growth, apoptosis regulation, cytokine signaling, and activation of immune cells (Tanaka et al., 1996; Nguyen et al., 1997; Tada et al., 1997; Taniguchi et al., 2001; Tamura et al., 1995, 2008). Different sets of genes are selectively modulated by IRF1 expression, which depends on cell type and/or cell stimuli to evoke appropriate responses (Taniguchi et al., 2001; Shi et al., 2008). For example, the IRF1 gene in pigs is overexpressed in endometrial cells during the peri-implantation period, which contributes to establishing successful pregnancy (Joyce et al., 2007). In goats, studies that examined immune response to mammary gland infection with Staphylococcus aureus showed a high increase in IRF1 gene expression compared with other genes that are related to innate resistance to pathogens (Cremonesi et al., 2012).

In Brazil, studies on the river buffalo genome started in 2007 with the construction of a whole genome mapping tool that was characterized by a radiation hybrid cell panel (Amaral et al., 2007). Recently, another genomic tool, a buffalo large-insert bacterial artificial chromosome (BAC) library, was developed for the whole buffalo genome. This library facilitated the validation and accurate assembly of genome sequences, isolation of genes and gene clusters, elucidation of gene organization, and identification of regulatory and repetitive elements (Stafuzza et al., 2012).

Currently, several research groups are using next-generation sequencing (NGS) platforms to produce genome sequences for various buffalo breeds (Michelizzi et al., 2010, Tantia et al., 2011). Earlier this year, a whole buffalo genome sequence from an individual of the Mediterranean breed was publically released in the National Center for Biotechnology Information (NCBI) database (http://www.ncbi.nlm.nih.gov/assembly/67671). Although NGS platforms have been used to produce buffalo genome sequences, recent studies of de novo assemblies that were produced by this approach have indicated a loss of approximately $16 \%$ of the genome (Alkan et al., 2011).

The IRF1 gene has previously been mapped on river buffalo chromosome 9 using radiation hybrid panel technology (Amaral et al., 2008). Considering that this gene could be an important candidate gene for studying disease susceptibility, we characterized its molecular structure in buffalo by sequencing a clone from the buffalo large-insert BAC library. A comparative analysis was performed using the Murrah and Mediterranean breeds to search for potential nucleotide variations. The coding and amino acid sequences were also compared against those of other vertebrates. 


\section{MATERIAL AND METHODS}

\section{BAC library screening}

The buffalo BAC library was three-dimensionally screened by PCR with the same cattle-derived primers that were used for radiation hybrid mapping of the IRF1 gene in the river buffalo chromosome 9 (forward 5'-GGGTCACACAGGTAGTCATCAT-3' and reverse 5'-ATGTGCTAGGACCCATACAGAG-3') (Everts-van der Wind et al., 2004).

The 3-D screening of the library was carried out in three steps. The first step was performed with 17 superpools, where each superpool contained BAC clones from eight 384 well plates. In the second step, eight single pools, which represented BAC clones from each of the eight plates in the positive superpool, were screened. Finally, the pooled row and column BAC clones from the positive plate were screened. The intersection of the row and column corresponded to the location of the positive BAC clone for the IRF1 gene.

Polymerase chain reactions (PCRs) were performed in a $25 \mu \mathrm{L}$ volume that contained $10 \mathrm{mM}$ Tris- $\mathrm{HCl}, 1.5 \mathrm{mM} \mathrm{MgCl}, 50 \mathrm{mM} \mathrm{KCl}, 10 \mathrm{mM}$ dNTPs, $0.2 \mathrm{mM}$ of each primer, 0.5 unit of AmpliTaq Gold polymerase (Life Technologies ${ }^{\mathrm{TM}}$, USA), and $2 \mu \mathrm{L}$ BAC DNA. The amplification conditions were carried out under the following conditions: initial denaturation at $94^{\circ} \mathrm{C}$ for $10 \mathrm{~min}$, followed by 35 cycles at $94^{\circ} \mathrm{C}$ for $30 \mathrm{~s}$ (denaturation), $65^{\circ} \mathrm{C}$ for $30 \mathrm{~s}$ (annealing), $72^{\circ} \mathrm{C}$ for $30 \mathrm{~s}$ (extension), and a final extension at $72^{\circ} \mathrm{C}$ for $7 \mathrm{~min}$. The PCR products were electrophoresed through $2 \%$ agarose gels in $1 \mathrm{X}$ TBE (Tris/Borate/EDTA) buffer containing $0.25 \mu \mathrm{g} / \mathrm{mL}$ ethidium bromide and photographed under UV light.

\section{Isolation of BAC DNA and sequencing}

The positive clone for the IRF1 gene was grown in $50 \mathrm{~mL}$ of Luria-Bertani medium (Sigma-Aldrich, USA) containing $12.5 \mu \mathrm{g} / \mathrm{mL}$ chloramphenicol. The BAC DNA was purified using the PhasePrep ${ }^{\mathrm{TM}}$ BAC DNA Kit (Sigma-Aldrich, USA) as described by the manufacturer. Briefly, clones were harvested by centrifugation at 5,000 $\mathrm{g}$ for $10 \mathrm{~min}$. Supernatant was removed, and the pellet was resuspended in $250 \mu \mathrm{L}$ of a solution containing RNase and $250 \mu \mathrm{L}$ of lysis components followed by the addition of $250 \mu \mathrm{L}$ of neutralization solution followed by incubation on ice for $5 \mathrm{~min}$ and centrifugation at $16,000 \mathrm{~g}$ for $5 \mathrm{~min}$ at $4^{\circ} \mathrm{C}$. The supernatant was transferred to 2 -mL microcentrifuge tubes, and $450 \mu \mathrm{L}$ of isopropanol was added. The nucleic acids were collected by centrifugation at $16,000 \mathrm{~g}$ for $20 \mathrm{~min}$ at $4^{\circ} \mathrm{C}$. The pellet was washed with $100 \mu \mathrm{L}$ of $70 \%$ ethanol and followed by addition of $500 \mu \mathrm{L}$ of elution solution.

The endotoxins and other impurities were removed by addition of $100 \mu \mathrm{L}$ of endotoxin removal solution followed by incubation on ice for $5 \mathrm{~min}$ and $37^{\circ} \mathrm{C}$ for $5 \mathrm{~min}$. The clear upper phase, which contained the BAC DNA, was transferred to another 2-mL microcentrifuge tube, followed by adding $540 \mu \mathrm{L}$ of DNA precipitation solution and centrifugation at 16,000 $g$ for $20 \mathrm{~min}$ at $4{ }^{\circ} \mathrm{C}$. The supernatant was removed and the pellet was washed with $150 \mu \mathrm{L}$ of $70 \%$ ethanol, followed by centrifugation at $16,000 \mathrm{~g}$ for $20 \mathrm{~min}$ at $4^{\circ} \mathrm{C}$. The supernatant was discarded and the BAC DNA was eluted in $100 \mu \mathrm{L}$ of Tris-EDTA buffer.

The DNA concentration was determined by NanoDrop spectrophotometer (Thermo Scientific, USA) and adjusted to $100 \mathrm{ng} / \mu \mathrm{L}$. An additional PCR with the purified DNA was performed as described earlier to confirm amplification of the IRF1 gene. 
The BAC DNA sequencing was obtained by NGS using 454-pyrosequencing with GS FLX Titanium chemistry (Roche, Germany) at the 454 Sequencing Center, Branford, CT, USA. The sequence data were assembled using the GS De Novo Assembler version 2.6 (Roche, Germany).

\section{Bioinformatic analysis}

Repetitive DNA elements in the Murrah BAC clone sequence were identified by RepeatMasker (http://www.repeatmasker.org/). The Augustus software was used to predict genes from the masked nucleotide sequence (http://bioinf.uni-greifswald.de/augustus/). The predicted gene sequence was aligned against NCBI reference mRNA sequences of Bos taurus using BLAST tools (http://blast.ncbi.nlm.nih.gov/Blast.cgi). Additionally, the predicted peptide sequence was aligned against non-redundant protein sequences by BLASTp (protein-protein BLAST) to verify homology with other species and identify the predicted gene.

The coordinates of exons, introns, 5'UTR, and 3'UTR were obtained by performing an alignment between the BAC sequence from the Murrah breed against the predicted gene sequence available for the Mediterranean breed on NCBI (http:/www.ncbi.nlm.nih.gov/assembly/67671). In addition, the Splign program (http://www.ncbi.nlm.nih.gov/sutils/splign/ splign.cgi) was used to recognize introns and splice signals.

VISTA plots were generated using wgVISTA alignment (http://genome.lbl.gov/cgibin/WGVistaInput) with the bovine chromosome 7 genomic scaffold (GenBank accession No. NW_003104019.1) to show the relative nucleotide conservation between buffalo and cattle. The Clustal Omega software (http://www.ebi.ac.uk/Tools/msa/clustalo/) was used to align coding gene sequences and amino acids sequences from buffalo (Bubalus bubalis), cattle (Bos taurus), yaks (Bos mutus), goats (Capra hircus), sheep (Ovis aries), horses (Equus caballus), humans (Homo sapiens), rats (Rattus norvegicus), mice (Mus musculus), and chickens (Gallus gallus).

\section{RESULTS}

Screening the buffalo BAC library resulted in the identification of one positive clone for the IRF1 gene. The 454/Roche GS FLX sequencer generated a total of 3973 reads with an average read length of 709 nucleotides that are arranged in one contig of 44,370 nucleotides, providing $61.18 \mathrm{X}$ coverage and $99.90 \%$ of Q40. The Murrah BAC DNA sequence was deposited in GenBank under accession No. KJ635887.

The gene prediction by the Augustus software detected one gene in the Murrah BAC sequence. The alignment of the predicted gene against the NCBI reference mRNA sequences from Bos taurus revealed $99 \%$ identity and $100 \%$ coverage with bovine IRF1 mRNA(GenBank accession No. NM_001191261.2). The predicted peptide alignment against non-redundant protein sequences from Bos taurus database revealed 100\% identity and 100\% coverage with the bovine IRF1 protein (GenBank accession No. NP 001178190.1).

The alignment between the Murrah BAC sequence against the predicted gene for the Mediterranean breed (GenBank accession No. NW_005783806.1, unplaced genomic scaffold scf7180021615926, UMD_CASPUR_WB_2.0) established that the coordinates of the IRF1 gene are from nucleotide 26,704 to nucleotide 33,802 , with a total length of $7099 \mathrm{bp}$, which includes 10 exons and nine introns. Figure 1 shows the exon/intron organization of the IRF1 gene in river buffalos and its counterpart in yaks, cattle, goats, horses, humans, mice, rats, and 
chickens. The organization of the gene was as follows: non-protein-coding exon 1 (244 bp), intron 1 (1036 bp), exon 2 (92 bp), intron 2 (1280 bp), exon 3 (100 bp), intron 3 (796 bp), exon 4 (177 bp), intron 4 (123 bp), exon 5 (50 bp), intron 5 (107 bp), exon 6 (133 bp), intron 6 (190 bp), exon 7 (123 bp), intron 7 (307 bp), exon 8 (50 bp), intron 8 (851 bp), exon 9 (136 bp), intron 9 (314 bp), exon 10 (113 bp), and 3'UTR (877 bp). The 5'UTR showed a total length of $249 \mathrm{bp}$ containing the total length of non-protein-coding exon 1 and $5 \mathrm{bp}$ from exon 2 . The IRF1 gene showed a exon/intron size ratio of $1: 4.10$ and the CG content of $54.04 \%$. The coding sequence showed a total of 969 nucleotides that encode the IRF1 protein with 322 amino acids.

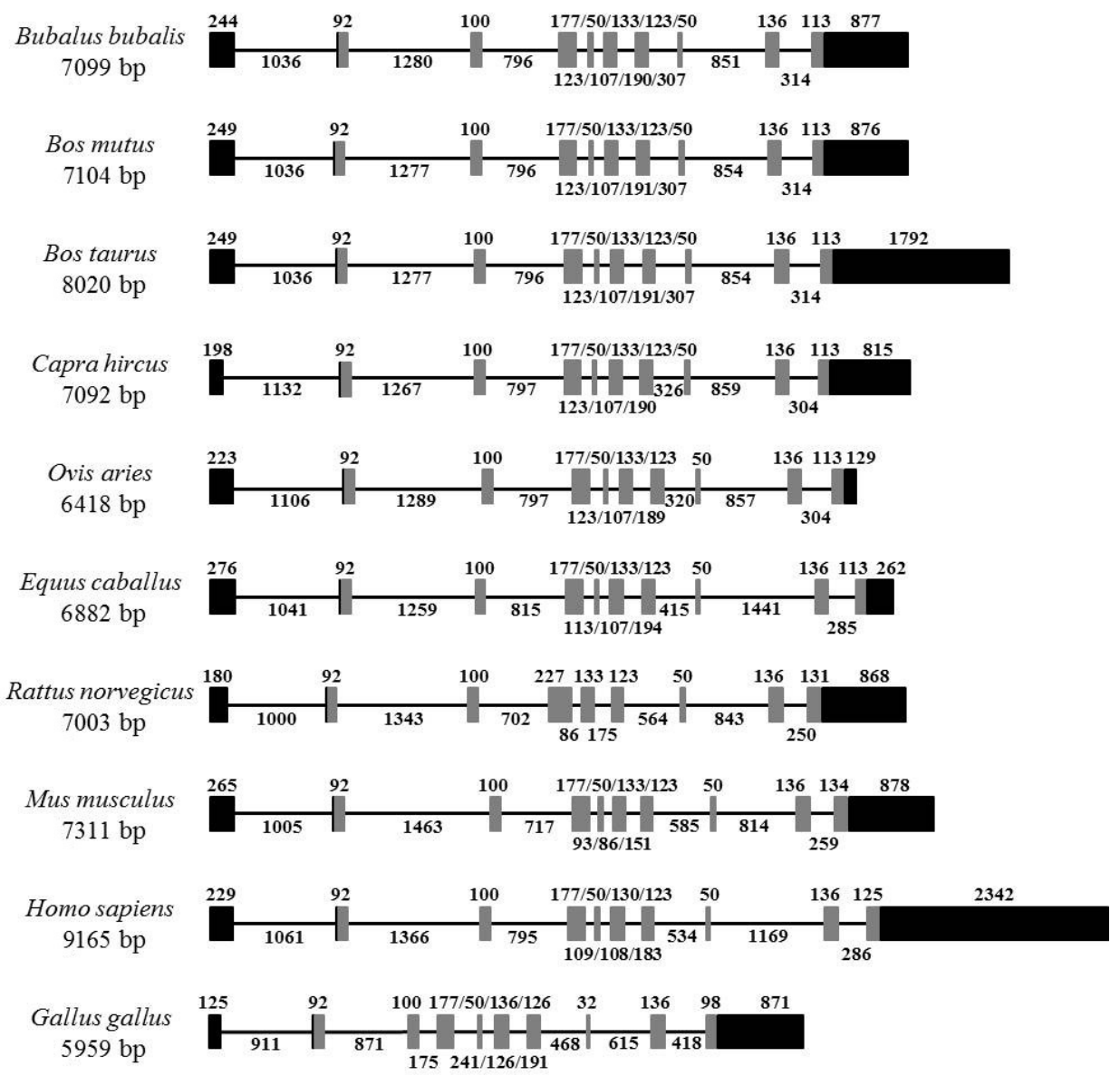

Figure 1. Schematic illustration of the exon/intron organization of the IRF1 gene in Bubalus bubalis, Bos mutus (GenBank accession No. XM_005888366.1), Bos taurus (GenBank accession No. NM_001191261.2), Capra hircus (GenBank accession No. XM_005682621.1), Ovis aries (GenBank accession No. NM_001009751.1), Equus caballus (GenBank accession No. XM_001504445.2), Rattus norvegicus (GenBank accession No. NM_012591.1), Mus musculus (GenBank accession No. NM_008390.2), Homo sapiens (GenBank accession No. NM_002198.2), and Gallus gallus (GenBank accession No. NM_205415.1). Exons are indicated by grey boxes while introns are indicated by black lines. The 5'UTR and 3'UTR are represented by black boxes. The size of the exons and the UTRs are shown above the gray and black boxes. The intron size is shown below the black lines. 
Alignment of the IRF1 gene sequence from the Murrah breed against the predicted gene sequence from the Mediterranean breed revealed 99\% identity, with six nucleotide substitutions and three deletions as follows: 5'UTR (g.122A $>$ C, g.126A $>$ G, and g.127A $>$ C), exon 4 (g.3676T $>$ C), intron 8 (g.5190delT and g.5191delT), and 3'UTR (g.6657delA, g. $6715 \mathrm{~A}>\mathrm{G}$, and g.6976C $>\mathrm{T})$.

Comparative analysis using the wgVISTA alignment between the IRF1 gene of buffalo and cattle (chromosome 7 genomic scaffold, GenBank accession no NW_003104019.1) showed higher than $97 \%$ genomic conservation. In addition, we observed a total of 12 gaps and 113 nucleotide substitutions with five found in coding regions (g.2720T $>$ C, g.3678T $>$ C, g.4064G $>$ T, g.5716T $>$ C, and g.5731T $>$ C).

The multiple alignment of the buffalo IRF1 coding sequence against other bovids, humans, horses, rodents, and chickens showed the following: 99\% sequence identity with cattle and yaks (GenBank accession no NM_001191261.2 and XM_005888366.1, respectively), 98\% with goats and sheep (GenBank accession no XM_005682621.1 and NM_001009751.1, respectively), 92\% with horses (GenBank accession no XM_001504445.2), 88\% with humans (GenBank accession no NM_002198.2), 83\% with mice and rats (GenBank accession no NM_008390.2 and NM_012591.1, respectively), and 73\% with chickens (GenBank accession no NM_205415.1). All alignments showed 100\% coverage, except rats and chickens, which had $99 \%$ and $72 \%$ coverage, respectively.

On the protein level, the amino acid sequence of the buffalo IRF1 protein revealed $100 \%$ identity with cattle and yaks (GenBank accession no NP_001178190.1 and XP_005888428.1, respectively), 98\% with goats and sheep (GenBank accession no XP_005682678.1 and NP_001009751.1, respectively), 90\% with horses (GenBank accession no XP_001504495.1), 88\% with humans (GenBank accession no NP_002189.1), 81\% with mice (GenBank accession No. NP_032416.1), 80\% with rats (GenBank accession no NP_036723.1), and 61\% with chickens (GenBank accession no NP_990746.1). All alignments showed $100 \%$ coverage, except for chickens, which had $99 \%$ coverage. Figure 2 shows a schematic representation of the amino acid sequence alignments, which indicates the protein domains previously described in humans and mice, such as DNA binding (1-120 aa; Schaper et al., 1998), N-terminal repressor (1-60 aa; Kirchhoff et al., 2000), nuclear localization (117-141 aa; Schaper et al., 1998), transactivation (233-255 aa; Kim et al., 2003), and enhancer domain (257-325 aa; Pion et al., 2009).

The RepeatMasker tool identified a CG content of $48.46 \%$ and a total of 67 repetitive elements in the Murrah BAC DNA sequence. Statistical analysis revealed that $41.46 \%$ of the buffalo DNA (18,394 bp) comprised a total of 59 interspersed repeats, which included 29 small interspersed nucleotide elements (SINEs), 22 long interspersed nucleotide elements (LINEs), two long terminal repeat elements (LTRs), and six DNA elements. Other repetitive elements included six small RNAs, one simple tandem repeat, one satellite DNA sequence, and one low-complexity DNA sequence. The repetitive elements are summarized in Table 1. The buffalo IRF1 gene showed the presence of only three repetitive elements including one L2c on intron 1, one MIR, and one THER1_MD on intron 2, which represents $6.20 \%$ of the total length of the gene. 


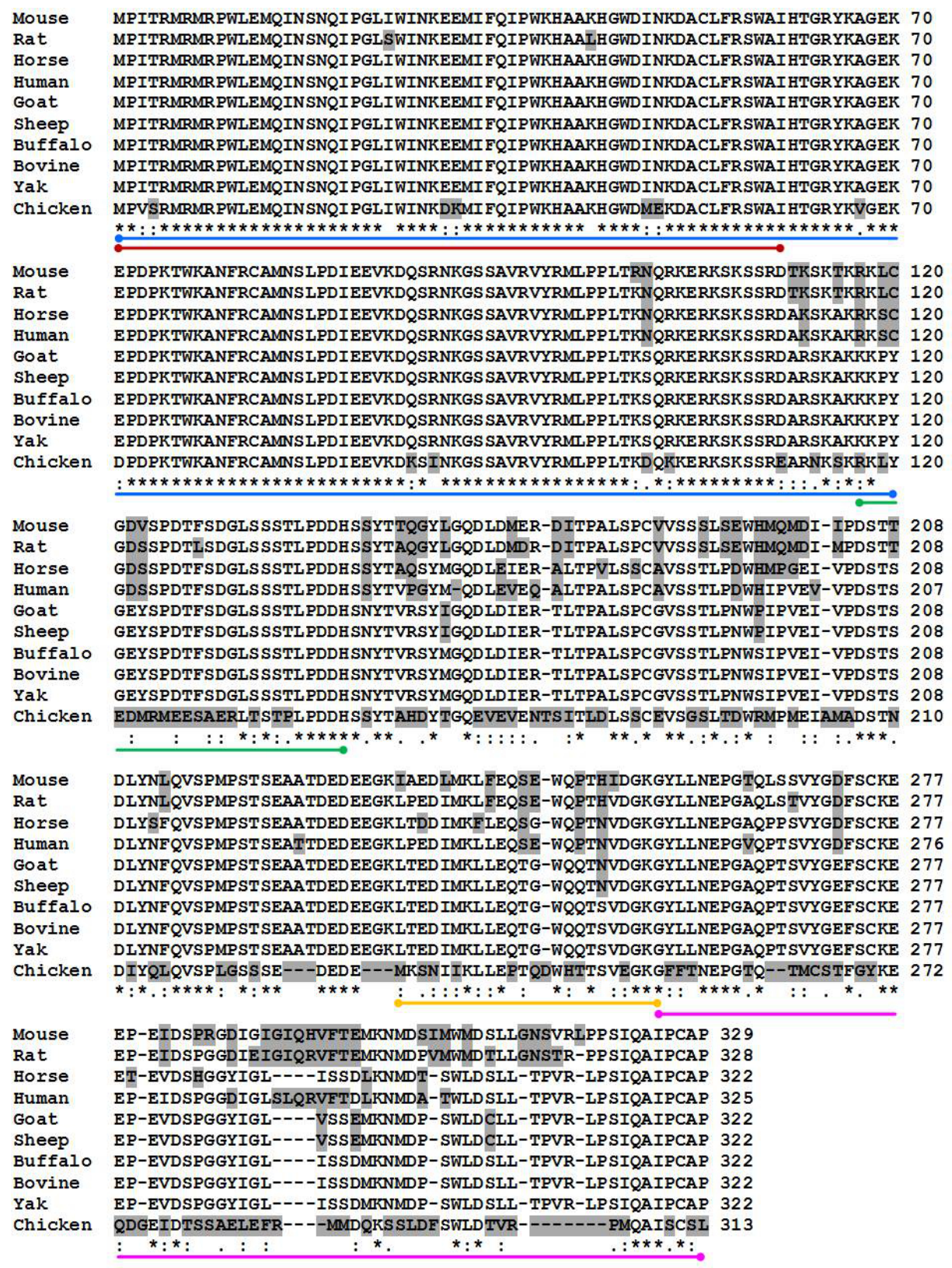

Figure 2. Multispecies alignment using the Clustal Omega software showing the percentage of identity between the buffalo interferon regulatory factor 1 amino acid sequence against its counterparts in mice, rats, horses, humans, goats, sheep, cattle, yaks, and chickens. Amino acid substitutions that are related to the buffalo sequence are highlighted in gray. The colored lines indicate the different domains of interferon regulatory factor 1 protein based on the coordinates in the human protein: the DNA binding domain is represented in blue (1-120 aa), N-terminal repressor domain in red (1-60 aa), nuclear localization domain in green (117-141 aa), transactivation domain in orange (233-255 aa), and enhancer domain in pink (255-325 aa). 
Table 1. Repetitive elements identified in the Murrah BAC DNA sequence. Data include type of repetitive element, the total number of repeats, the total length (bp), and the percentage of each element observed in the BAC DNA sequence.

\begin{tabular}{lccc}
\hline Type of element & $\mathrm{N}$ & Total length (bp) & DNA sequence (\%) \\
\hline Interspersed repeats & 59 & 18,394 & 41.46 \\
SINEs & 29 & 4,023 & 9.07 \\
LINEs & 22 & 12,948 & 29.18 \\
LTRs & 2 & 560 & 1.26 \\
DNA elements & 6 & 863 & 1.95 \\
Small RNA & 6 & 909 & 2.05 \\
Satellite & 1 & 72 & 0.16 \\
Simple repeat & 1 & 196 & 0.44 \\
Low complexity DNA & 1 & 34 & 0.08 \\
\hline
\end{tabular}

\section{DISCUSSION}

In this study, one BAC clone from the buffalo BAC library was isolated, characterized, and showed the complete sequence of the IRF1 gene, with a total size of $7099 \mathrm{bp}$. The highly conserved organization and size of the IRF1 gene was evident when we observed the number and sizes of the exons and introns in different species of bovids, rodents, horses, humans, and chickens (Figure 1). All species showed the same number of exons (10) and introns (nine), except for rats, which did not have exon 5, but had the largest exon 4, with $227 \mathrm{bp}$ instead of $177 \mathrm{bp}$. This suggests that the exon 5 was incorporated into exon 4.

The main difference observed on the IRF1 gene was regarding the size of the 3'UTR. Among the members of the Bovidae family, buffalo, yaks, and goats presented a 3'UTR of almost the same size (877, 876, and $815 \mathrm{bp}$, respectively). Alternatively, cattle showed a 3'UTR that was approximately twice the size of that of buffalo, yaks, and goats, with a total size of 1792 bp. Comparison between the gene sequences from buffalo and cattle using wgVISTA showed $97.3 \%$ genomic conservation of the $3^{\prime} \mathrm{UTR}$, and no repetitive elements were observed in the conserved region. In contrast, sheep presented a smaller 3 'UTR region (123 bp) compared with all species analyzed, while human showed the largest 3'UTR with $2342 \mathrm{bp}$. Regarding the 5'UTR region, similar sizes and structure were observed among all species and always included the entire non-protein-coding exon 1 as well as $5 \mathrm{bp}$ from exon 2 (Figure 1).

Despite the differences observed in the total size of the IRF1 gene among species, the size of the coding sequences remained the same in bovids and horses ( $969 \mathrm{bp})$. Chickens had a smaller coding sequence (942 bp), while mice (990 bp) had the largest. Considering that the IRF1 amino acid sequences from buffalo, cattle, and yaks showed $100 \%$ identity, it is possible to extrapolate that the protein could be associated with the same functional role in these three species. Additionally, the multispecies alignment demonstrated that the protein DNA-binding domain (1-120 aa) is the most conserved region of the protein, while the enhancer domain (255-325 aa) was less conserved (Figure 2).

Regarding the diversity of the repetitive elements in the IRF1 gene, we observed the presence of five categories of elements in the bovids, including SINEs (five), a LINE (one), DNA elements (two), simple repeats (three), and a satellite DNA sequence (one). SINE elements are the most common and were present in all bovids. In buffalo, we observed the occurrence of three elements, two SINEs (MIR and THER1_MD) and one LINE (Lc2). Yaks presented only two SINEs while cattle showed the higher number of elements (six). Additionally, the bovine gene was the only one with the presence of repetitive elements in the 3'UTR, 
but the location of the elements were exclusively in the bovine-specific portion of the region.

Comparative analysis between the gene sequences from two different buffalo breeds, Murrah (our sequence) and Mediterranean (publicly available at NCBI), revealed six single nucleotide variations (potential single nucleotide polymorphisms, SNPs). According to previous literature, most SNPs are located in non-protein-coding regions of the gene, such as introns, as well as $5^{\prime}$ and $3^{\prime}$ UTRs. Interestingly, the buffalo IRF1 gene showed most of the nucleotide substitutions in the $5^{\prime}$ and $3^{\prime}$ UTRs (five). Intron 8 was the only region that contained two single nucleotide deletions (g.5190delT and g.5191delT), which is evidence of a high level of DNA sequence conservation in the structure of this gene.

\section{ACKNOWLEDGMENTS}

Research supported by Fundação de Amparo à Pesquisa do Estado de São Paulo (FAPESP) (grant \#2011/11889-3) to M.E.J. Amaral-Trusty and a post-doctoral fellowship to N.B. Stafuzza (fellowship \#2011/02478-0). Coordenação de Aperfeiçoamento de Pessoal de Nível Superior) funded a Master's fellowship to M.M. Borges.

\section{REFERENCES}

Alkan C, Sajjadian S and Eichler EE (2011). Limitations of next-generation genome sequence assembly. Nat. Methods 8: 61-65.

Amaral ME, Owens KE, Elliott JS, Fickey C, et al. (2007). Construction of a river buffalo (Bubalus bubalis) wholegenome radiation hybrid panel and preliminary RH mapping of chromosomes 3 and 10. Anim. Genet. 38: 311-314.

Amaral ME, Grant JR, Riggs PK, Stafuzza NB, et al. (2008). A first generation whole genome RH map of the river buffalo with comparison to domestic cattle. BMC Genomics 9: 631.

Bazer FW, Burghardt RC, Johnson GA, Spencer TE, et al. (2008). Interferons and progesterone for establishment and maintenance of pregnancy: interactions among novel cell signaling pathways. Reprod. Biol. 8: 179-211.

Chen W and Royer Jr WE (2010). Structural insights into interferon regulatory factor activation. Cell Signal. 22: 883-887.

Cremonesi P, Capoferri R, Pisoni G, Del Corvo M, et al. (2012). Response of the goat mammary gland to infection with Staphylococcus aureus revealed by gene expression profiling in milk somatic and white blood cells. BMC Genomics 13: 540 .

Everts-van der Wind A, Kata SR, Band MR, Rebeiz M, et al. (2004). A 1463 gene cattle-human comparative map with anchor points defined by human genome sequence coordinates. Genome Res. 14: 1424-1437.

Fragale A, Marsili G and Battistini A (2013). Genetic and epigenetic regulation of interferon regulatory factor expression: implications in human malignancies. J. Genet. Syndr. Gene Ther. 4: 11.

Honda $\mathrm{K}$ and Taniguchi T (2006). IRFs: master regulators of signaling by Toll-like receptors and cytosolic patternrecognition receptors. Nat. Rev. Immunol. 6: 644-658.

Joyce MM, Burghardt JR, Burghardt RC, Hooper RN, et al. (2007). Pig conceptuses increase uterine interferon-regulatory factor 1 (IRF1), but restrict expression to stroma through estrogen-induced IRF2 in luminal epithelium. Biol. Reprod. 77: 292-302.

Kim EJ, Park CH, Park JS and Um SJ (2003). Functional dissection of the transactivation domain of interferon regulatory factor-1. Biochem. Biophys. Res. Commun. 304: 253-259.

Kirchhoff S, Oumard A, Nourbakhsh M, Levi BZ, et al. (2000). Interplay between repressing and activating domains defines the transcriptional activity of IRF-1. Eur. J. Biochem. 267: 6753-6761.

Mamane Y, Heylbroeck C, Génin P, Algarté M, et al. (1999). Interferon regulatory factors: the next generation. Gene 237: $1-14$.

Michelizzi VN, Dodson MV, Pan Z, Amaral ME, et al. (2010). Water buffalo genome science comes of age. Int. J. Biol. Sci. 6: 333-349.

Nehyba J, Hrdlicková F and Bose HR (2009). Dynamic evolution of immune system regulators: the history of the interferon regulatory factor family. Mol. Biol. Evol. 26: 2539-2550.

Nguyen H, Hiscott J and Pitha PM (1997). The growing family of interferon regulatory factors. Cytokine Growth Factor Rev. 8: 293-312.

Genetics and Molecular Research 14 (3): 10919-10928 (2015)

CFUNPEC-RP www.funpecrp.com.br 
Ozato K, Tailor P and Kubota T (2007). The interferon regulatory factor family in host defense: mechanism of activation. J. Biol. Chem. 282: 20065-20069.

Pion E, Narayan V, Eckert M and Ball KL (2009). Role of the IRF-1 enhancer domain in signaling polyubiquitination and degradation. Cell Signal. 21: 1479-1487.

Schaper F, Kirchhoff S, Posern G, Köster M, et al. (1998). Functional domains of interferon regulatory factor I (IRF-1). Biochem. J. 335: 147-157.

Shi Y, Zhang YB, Zhao Z, Jiang J, et al. (2008). Molecular characterization and subcellular localization of Carassius auratus interferon regulatory factor-1. Dev. Comp. Immunol. 32: 134-146.

Stafuzza NB, Abbey CA, Gill CA, Womack JE, et al. (2012). Construction and preliminary characterization of a river buffalo bacterial artificial chromosome library. Genet. Mol. Res. 11: 3013-3019.

Tada Y, Ho A, Matsuyama T and Mak TW (1997). Reduced incidence and severity of antigen-induced autoimmune diseases in mice lacking interferon regulatory factor-1. J. Exp. Med. 185: 231-238.

Tamura T, Ishihara M, Lamphier MS, Tanaka N, et al. (1995). An IRF-1-dependent pathway of DNA damage-induced apoptosis in mitogen-activated T lymphocytes. Nature 376: 596-599.

Tamura T, Yanai H, Savitsky D and Taniguchi T (2008). The IRF family transcription factors in immunity and oncogenesis. Annu. Rev. Immunol. 26: 535-584.

Tanaka N, Ishihara M, Lamphier MS, Nozawa H, et al. (1996). Cooperation of the tumour suppressors IRF-1 and p53 in response to DNA damage. Nature 382: 816-818.

Taniguchi T, Ogasawara K, Takaoka A and Tanaka N (2001). IRF family or transcription factors as regulators of host defense. Annu. Rev. Immunol. 19: 623-655.

Tantia MS, Vijh RK, Bhasin V, Sikka P, et al. (2011). Whole-genome sequence assembly of the water buffalo (Bubalus bubalis). Indian J. Anim. Sci. 81: 38-46. 